\title{
Broadband Impedance Transformer Based on Asymmetric Coupled Transmission Lines in Nonhomogeneous Medium
}

\author{
Zhurbenko, Vitaliy; Krozer, Viktor; Meincke, Peter
}

Published in:

International Microwave Symposium Digest

Link to article, DOI:

10.1109/MWSYM.2007.380142

Publication date:

2007

Document Version

Publisher's PDF, also known as Version of record

Link back to DTU Orbit

Citation (APA):

Zhurbenko, V., Krozer, V., \& Meincke, P. (2007). Broadband Impedance Transformer Based on Asymmetric Coupled Transmission Lines in Nonhomogeneous Medium. In International Microwave Symposium Digest (pp. 1893-1896). IEEE. https://doi.org/10.1109/MWSYM.2007.380142

\section{General rights}

Copyright and moral rights for the publications made accessible in the public portal are retained by the authors and/or other copyright owners and it is a condition of accessing publications that users recognise and abide by the legal requirements associated with these rights.

- Users may download and print one copy of any publication from the public portal for the purpose of private study or research.

- You may not further distribute the material or use it for any profit-making activity or commercial gain

- You may freely distribute the URL identifying the publication in the public portal 


\title{
Broadband Impedance Transformer Based on Asymmetric Coupled Transmission Lines in Nonhomogeneous Medium
}

\author{
Vitaliy Zhurbenko, Viktor Krozer, Peter Meincke \\ Technical University of Denmark, Ørsted•DTU, Electromagnetic Systems, Kgs. Lyngby, \\ Ørsteds Plads, building 348, 2800, Denmark, vz@oersted.dtu.dk
}

\begin{abstract}
A new broadband quarter-wavelength impedance transformer based on an asymmetric coupled line section is presented. The bandwidth of the coupled line transformer is extended with the help of an interconnecting transmission line. An analytical model for the transformer is developed. The analysis of the structure reveals that a fractional bandwidth of more than $100 \%$ at $-20 \mathrm{~dB}$ reflection level can be achieved with such a structure. An experimental transformer circuit has been designed, fabricated and tested. Theoretical and experimental results are fair agreement and confirm the established theory. The achieved bandwidth is almost 3 times larger as compared with standard matching circuits.
\end{abstract}

Index Terms - Coupled transmission lines, impedance matching, impedance matrix, microstrip lines.

\section{INTRODUCTION}

Impedance matching components are fundamental elements in RF and microwave devices. State-of-the-art microwave systems always necessitate low cost and high performance well matched components. For highly compact microwave systems operating at low microwave frequencies employing traditional multiple quarter-wavelength transmission lines for wideband impedance matching becomes impractical. The same problem appears with matching of individual antenna elements in a large antenna array, when the available space for the feeding and matching networks is scarce. The resulting transformer length becomes a critical parameter for the design of miniature impedance matching circuit.

In recent years, symmetric coupled lines have been suggested as a matching element. These have the advantage for greater flexibility and compactness [1-3].

Symmetric coupled lines represent a restricted configuration of the more general class of coupled lines. They allow for a simpler analysis, however, for wideband applications asymmetric coupled lines are preferable. For example, the bandwidth of a forward-wave directional coupler using asymmetric coupled transmission lines is greater than the one formed using symmetric ones [4].

In this paper the design of a novel wideband impedance transformer based on asymmetric coupled lines is presented. In section II a general analytical model is derived for diagonally excited asymmetric coupled lines in nonhomogeneous dielectric medium. The model is helpful in analyzing the improvement in matching characteristics in comparison to standard inter-digital configuration. Section $\Pi I$ provides details of the proposed circuit with some examples and section IV reports experimental and theoretical results for the exemplified components.

\section{ASYMMETRIC COUPLED LINE SECTION IN NONHOMOGENEOUS DIELECTRIC MEDIUM}

The proposed wideband impedance transformer is based on asymmetric, uniform coupled lines in nonhomogeneous medium. A microstrip line is one of the most commonly used classes of transmission lines in nonhomogeneous medium. Edge-coupled microstrip lines are shown in Fig. 1. For the purpose of analysis, this coupled line four-port is transformed to a two-port network with arbitrary load using impedance matrix representation.

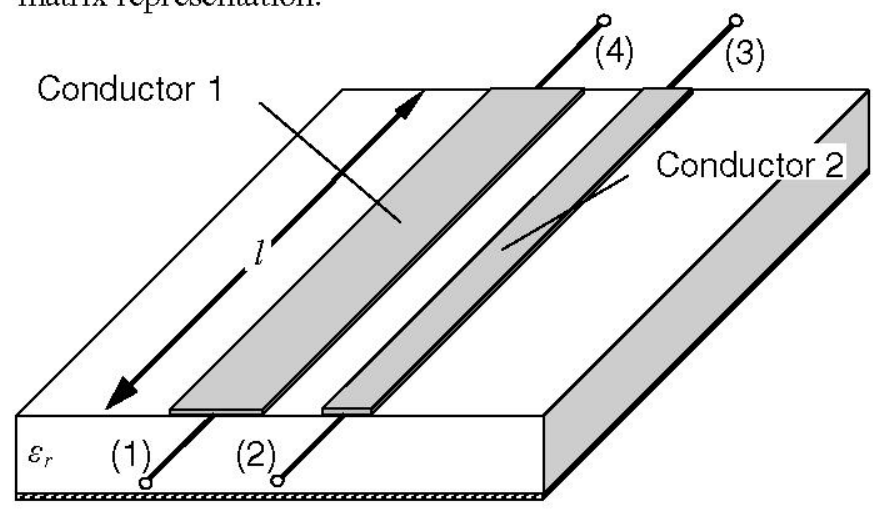

Fig. 1. A coupled microstrip line four-port.

The investigations presented in this paper are only for the most commonly used configuration, when diagonal terminals of the coupled lines are loaded with generator and load impedances. Thus, the entire circuit can be represented as a two-port network, which performs impedance transformation between a generator impedance $Z_{\mathrm{g}}$ connected to port 1 and a load impedance $Z_{\mathrm{L}}$ connected to port 3, as shown in Fig. 2.

As it can be seen in Fig. 2, the network consists of the coupled line four-port network described by an impedance matrix $[Z]$ and arbitrary load matrix at opposite terminals described by matrix $\left[Z^{\prime \prime}\right]$. In practice, ports 2 and 4 are in general either short-circuited or open-circuited with a corresponding representation of the two-port network [ $\left.Z^{\prime}\right]$.

The magnitude of the reflection coefficient at port 1 is equal to 


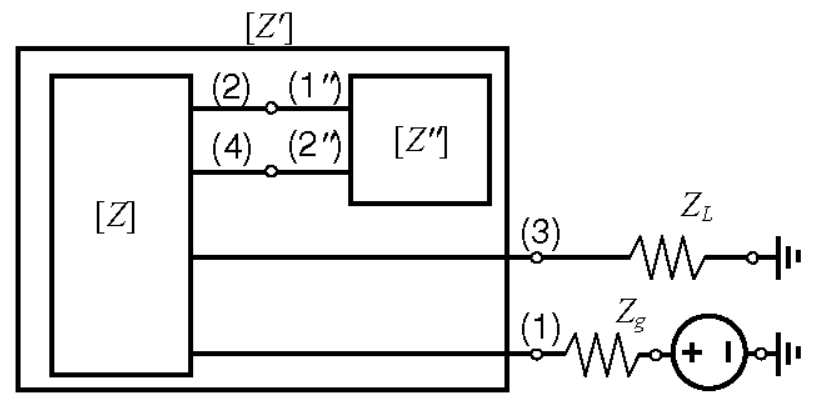

Fig. 2. Two-port network representation for the coupled line impedance transformer.

$$
\left|S_{11}\right|_{\mathrm{dB}}=20 \log \left(\left|\frac{Z_{i n}\left(Z_{i j}, Z_{i j}^{\prime \prime}, Z_{L}\right)-Z_{g}}{Z_{\text {in }}\left(Z_{i j}, Z_{i j}^{\prime \prime}, Z_{L}\right)+Z_{g}}\right|\right) \text {, }
$$

where $Z_{\text {in }}$ is the input impedance of the transformer, which is a function of the load impedance $Z_{\mathrm{L}}$, impedance matrix elements of coupled lines $Z_{\mathrm{ij}}$ and the arbitrary load $Z_{i j}^{\prime \prime}$ ( $i$ and $j$ are the indexes of matrix elements). Using the general impedance matrix representation for coupled lines [5] and boundary conditions at ports 2 and 4 the input impedance is expressed by

$$
Z_{\text {in }}=Z_{11}+Z_{12} \cdot a_{1}+Z_{14} \cdot b_{1}-\frac{\left(Z_{13}+Z_{12} \cdot a_{2}+Z_{14} \cdot b_{2}\right)^{2}}{Z_{33}+Z_{32} \cdot a_{2}+Z_{34} \cdot b_{2}+Z_{\mathrm{L}}},(2)
$$

where

$$
\begin{gathered}
a_{1}=\frac{Z_{41}\left(Z_{24}+Z_{12}^{\prime \prime}\right)-Z_{21}\left(Z_{44}+Z_{22}^{\prime \prime}\right)}{\left(Z_{22}+Z_{11}^{\prime \prime}\right)\left(Z_{44}+Z_{22}^{\prime \prime}\right)-\left(Z_{24}+Z_{12}^{\prime \prime}\right)\left(Z_{42}+Z_{21}^{\prime \prime}\right)} \\
a_{2}=\frac{Z_{43}\left(Z_{24}+Z_{12}^{\prime \prime}\right)-Z_{23}\left(Z_{44}+Z_{22}^{\prime \prime}\right)}{\left(Z_{22}+Z_{11}^{\prime \prime}\right)\left(Z_{44}+Z_{22}^{\prime \prime}\right)-\left(Z_{24}+Z_{12}^{\prime \prime}\right)\left(Z_{42}+Z_{21}^{\prime \prime}\right)}, \\
b_{1}=-\frac{Z_{41}}{\left(Z_{44}+Z_{22}^{\prime \prime}\right)}-\frac{\left(Z_{42}+Z_{21}^{\prime \prime}\right)}{\left(Z_{44}+Z_{22}^{\prime \prime}\right)} \cdot a_{1} \\
b_{2}=-\frac{Z_{43}}{\left(Z_{44}+Z_{22}^{\prime \prime}\right)}-\frac{\left(Z_{42}+Z_{21}^{\prime \prime}\right)}{\left(Z_{44}+Z_{22}^{\prime \prime}\right)} \cdot a_{2}
\end{gathered}
$$

A total number of six quantities is required to describe asymmetric coupled lines [4], being: $Z_{c l}$ and $Z_{\pi l}$, which are, respectively, the characteristic impedances of line 1 for $c$ and $\pi$ modes of propagation; $\gamma_{c}$ and $\gamma_{\pi}$, the propagation constants of $c$ and $\pi$ modes; $R_{c}$ and $R_{\pi}$, the ratios of the voltages on the two lines for $c$ and $\pi$ modes. Thus, the elements of the impedance matrix are given by

$$
Z_{11}=Z_{44}=\frac{Z_{c 1} \operatorname{coth}\left(\gamma_{c} l\right)}{1-R_{c} / R_{\pi}}+\frac{Z_{\pi 1} \operatorname{coth}\left(\gamma_{\pi} l\right)}{1-R_{\pi} / R_{c}},
$$

$$
\begin{aligned}
& Z_{12}=Z_{21}=Z_{34}=Z_{43}=\frac{Z_{c 1} R_{c} \operatorname{coth}\left(\gamma_{c} l\right)}{1-R_{c} / R_{\pi}}+\frac{Z_{\pi 1} R_{\pi} \operatorname{coth}\left(\gamma_{\pi} l\right)}{1-R_{\pi} / R_{c}} \\
& Z_{13}=Z_{31}=Z_{24}=Z_{42}=\frac{Z_{c 1} R_{c} \operatorname{csch}\left(\gamma_{c} l\right)}{\left(1-R_{c} / R_{\pi}\right)}+\frac{Z_{\pi 1} R_{\pi} \operatorname{csch}\left(\gamma_{\pi} l\right)}{\left(1-R_{\pi} / R_{c}\right)} \\
& Z_{14}=Z_{41}=\frac{Z_{c 1} \operatorname{csch}\left(\gamma_{c} l\right)}{\left(1-R_{c} / R_{\pi}\right)}+\frac{Z_{\pi 1} \operatorname{csch}\left(\gamma_{\pi} l\right)}{\left(1-R_{\pi} / R_{c}\right)} \\
& Z_{22}=Z_{33}=\frac{Z_{c 1} R_{c}^{2} \operatorname{coth}\left(\gamma_{c} l\right)}{1-R_{c} / R_{\pi}}+\frac{Z_{\pi 1} R_{\pi}^{2} \operatorname{coth}\left(\gamma_{\pi} l\right)}{1-R_{\pi} / R_{c}}, \\
& Z_{23}=Z_{32}=\frac{Z_{c 1} R_{c}^{2} \operatorname{csch}\left(\gamma_{c} l\right)}{\left(1-R_{c} / R_{\pi}\right)}+\frac{Z_{\pi 1} R_{\pi}^{2} \operatorname{csch}\left(\gamma_{\pi} l\right)}{\left(1-R_{\pi} / R_{c}\right)}
\end{aligned}
$$

where $l$ is the length of the coupled line section, as it is shown in Fig. 1. These relations are substituted into (3) and (2) to calculate the input impedance and finally the reflection coefficient of the transformer.

From relation (1) it can be seen that the matching properties of the transformer depend not only on coupled line parameters, but also on load of ports 2 and 4 , which are described by elements $Z_{i j}^{\prime \prime}$. This dependence introduces additional degree of freedom during design procedure and can be used to expand the bandwidth of the impedance transformer, as shown below.

\section{LOADING WITH TRANSMISSION LINE}

\section{A. Transmission Lines in Nonhomogeneous Medium}

As an example, terminals 2 and 4 are loaded with a microstrip transmission line. The impedance matrix of the transmission line with characteristic impedance $Z_{0}$, length $l$, and propagation constant $\gamma$ is given by

$$
\left[Z^{\prime \prime}\right]=\left[\begin{array}{cc}
Z_{0} \operatorname{coth}(\gamma l) & \frac{Z_{0}}{\sinh (\gamma l)} \\
\frac{Z_{0}}{\sinh (\gamma l)} & Z_{0} \operatorname{coth}(\gamma l)
\end{array}\right]
$$

The transformer configuration is shown in Fig. 3. In order to simplify further calculations, the transmission lines are considered to be lossless, and electrical lengths of the coupled line section $\left(\theta_{\mathbf{c}}+\theta_{\pi}\right) / 2$ and the microstrip transmission line $\theta$ are assumed equal, resulting in

$$
\begin{gathered}
\gamma l=j \beta l=j \theta, \quad \gamma_{\mathrm{c}} l=j \theta_{\mathrm{c}}, \quad \gamma_{\pi} l=j \theta_{\pi}, \\
\theta=\left(\theta_{\mathbf{c}}+\theta_{\pi}\right) / 2,
\end{gathered}
$$




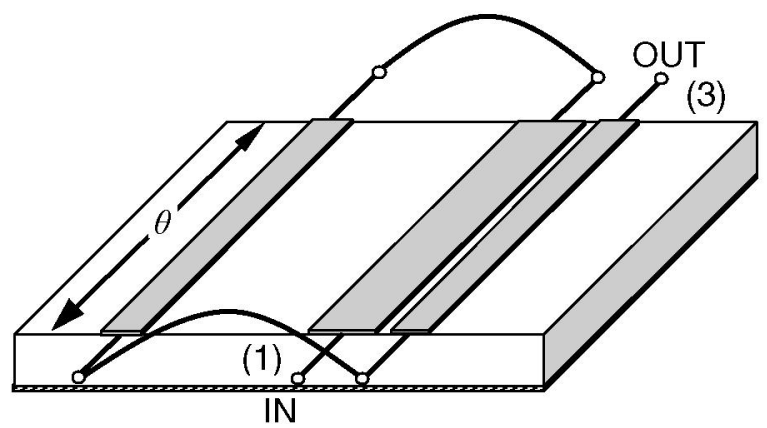

Fig. 3. Schematic illustration of the transformer based on coupled line section and a transmission line load.

where $\theta_{\mathrm{c}}$ and $\theta_{\pi}$ are the electrical lengths of the coupled line section for $c$ and $\pi$ mode respectively. $\theta$ is a function of frequency and can be used for the analysis of the spectrum of the transformer reflection coefficient. The calculated response (1) for the transformer of Fig. 3 is shown in Fig. 4.

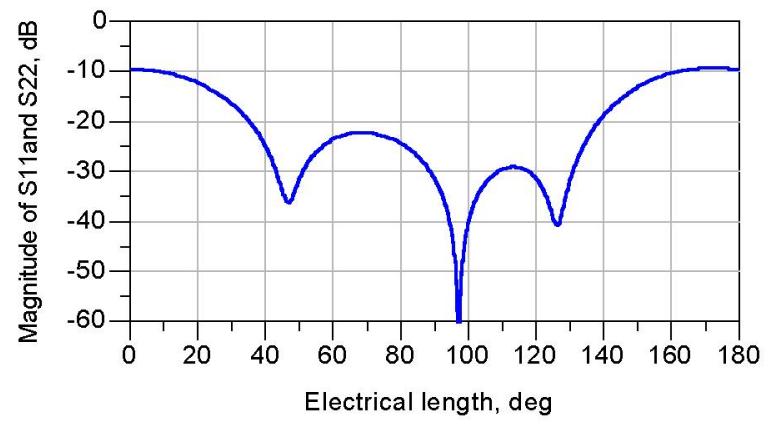

Fig. 4. Calculated reflection coefficient of transformer shown in Fig. 3. The transformation ratio is $1: 2$.

As it can be seen in Fig. 4 this transformer configuration exhibits an additional minimum in the reflection coefficient in comparison to the traditional impedance transformer based on coupled line section with open-circuited terminals [6]. These minima are non-uniformly distributed in the frequency domain. This is due to the differences in electrical lengths between the coupled line modes $\theta_{\mathrm{c}}$ and $\theta_{\pi}$ in nonhomogeneous medium.

\section{B. Comparison to Homogeneous Medium Case}

For the case of homogeneous medium the propagation constants for the two modes are equal, $\gamma_{\mathrm{c}}=\gamma_{\pi}$, and hence the electrical lengths for the two propagating modes are also equal. It is therefore possible to obtain three equidistant reflection zeros in the spectrum of the reflection coefficient. Because transmission lines in a homogeneous medium are a special case of transmission lines in a nonhomogeneous medium the expressions given in section II can also be used here for response calculations.

It can be depicted from the calculated response in Fig. 5 that the transformer provides wideband operation with uniformly distributed reflection zeros in the frequency domain. In addition, the distance between the zero locations can be varied by adjusting the parameters of the structure.

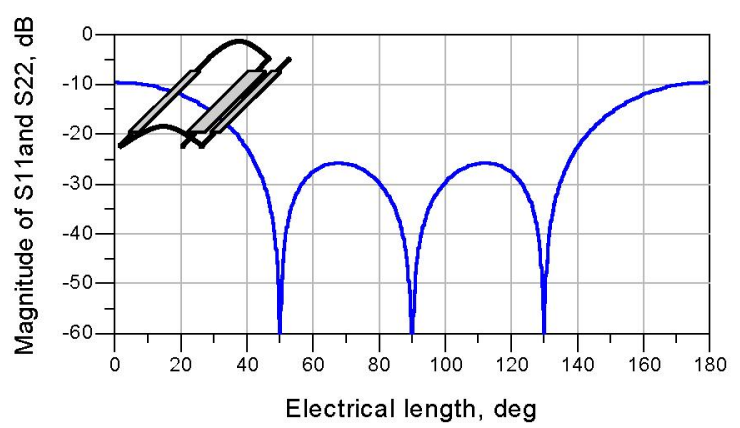

Fig. 5. Calculated reflection coefficient of the transformer for homogeneous medium case.

The electrical length of the transformer is equal to a quarter wavelength at the center frequency. Comparing the results in Fig. 4 and Fig. 5 it can be deduced that the impedance transformer in nonhomogeneous medium has approximately the same bandwidth as the one in homogenous medium. However, in many cases, like for example in surface mount technology, it is more useful to deal with microstrip structures.

\section{Design Example}

A matching circuit design example has been fabricated, based on a circuit configuration shown in Fig. 3. The center frequency is chosen to be $1 \mathrm{GHz}$ for convenience. At this frequency the electrical length of the microstrip structure is equal to a quarter wavelength on the line. A photograph of the fabricated $50-100 \Omega$ transformer is shown in Fig. 6. In this example the input transmission line is connected using an airbridge transition.

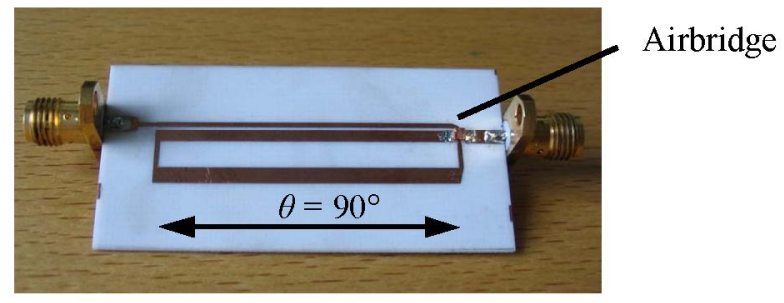

Fig. 6. Wideband quarter-wavelength impedance transformer. The microwave realization of the circuit in Fig. 3.

This matching circuit was implemented on a substrate with a dielectric constant $\varepsilon_{\mathrm{r}}=3.38$ and thickness $\mathrm{h}=0.8 \mathrm{~mm}$. The coupled line width is $1.39 \mathrm{~mm}$ for the input terminal and $0.56 \mathrm{~mm}$ for the output terminal. A transmission line width and gap between coupled lines are $1.93 \mathrm{~mm}$ and $0.6 \mathrm{~mm}$, respectively. The physical length of the transformer is $43.5 \mathrm{~mm}$. Calculated and measured results for this transformer are shown in Fig. 7. The measured fractional bandwidth for this configuration is more than $105 \%$ and $170 \%$ for $-20 \mathrm{~dB}$ and $-10 \mathrm{~dB}$ reflection coefficient level. For reference, the fractional bandwidth of the traditional quarter wave transformer is about $37 \%$ for $-20 \mathrm{~dB}$ reflection coefficient level. 


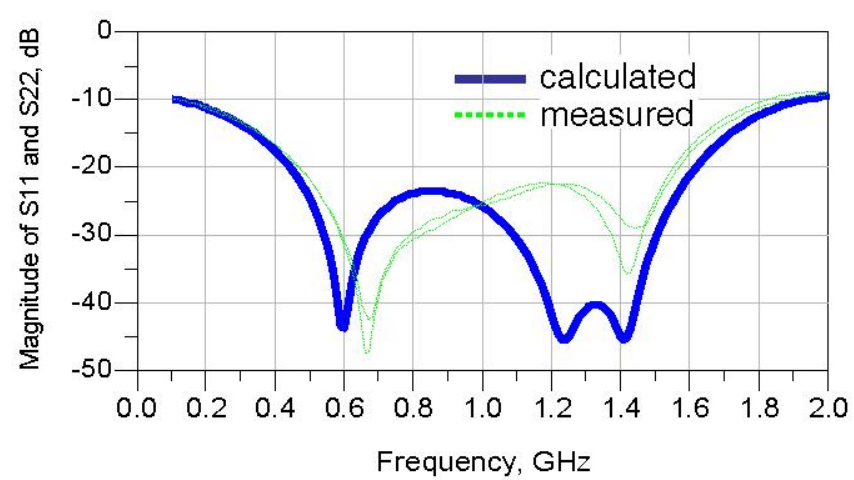

Fig. 7. Calculated and measured response for the transformer shown in Fig. 6.

The fractional bandwidth is defined as

$$
\delta f=\Delta f / f_{0}=2\left(f_{\mathrm{h}}-f_{1}\right) /\left(f_{\mathrm{h}}+f_{1}\right)
$$

where $f_{1}$ and $f_{\mathrm{h}}$ are the low and high end of the frequency band.

Differences between calculated and measured results at low magnitude of the reflection coefficient and at higher frequencies region are caused by fabrication inaccuracies, and a better agreement can be achieved by reducing the fabrication tolerances.

\section{LOADING WITH STEPPED IMPEDANCE TRANSMISSION LINE}

The differences in electrical lengths of the coupled lines in nonhomogeneous medium can be compensated by introducing a stepped impedance transmission line instead of the regular transmission line discussed in section III. For example, using two transmission lines with characteristic impedances $Z_{01}, Z_{02}$, and electrical lengths equal to $\theta / 2$, as it is shown in Fig. 8(a), it is possible to achieve a uniform distribution of reflection minima as indicated in Fig. 8(b).

Equation (1) was solved numerically for this transformer configuration with respect to the design parameters, taking into account the corresponding $\left[Z^{\prime \prime}\right]$ matrix representation. Based on these solutions, design curves for the transformer in Fig. 8(a) have been obtained.

\section{Conclusion}

It is shown that asymmetric, uniform coupled lines in nonhomogeneous dielectric medium, are an attractive component for wideband and compact impedance transformer design. It is demonstrated theoretically and experimentally that it is possible to improve the matching fractional bandwidth beyond $100 \%$ at $-20 \mathrm{~dB}$ reflection level by careful choice of the loads at the remaining terminals of the transformer. A general model for such a configuration of the transformer was developed based on mode characteristics. This general model establishes the design equations for the impedance transformer. Based on the analysis of this model different load configurations at the free terminals are proposed resulting in improved matching characteristics of the overall circuit.

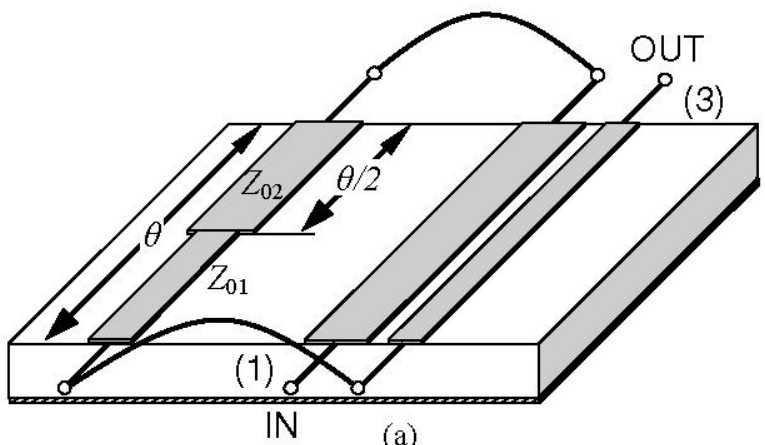

(a)

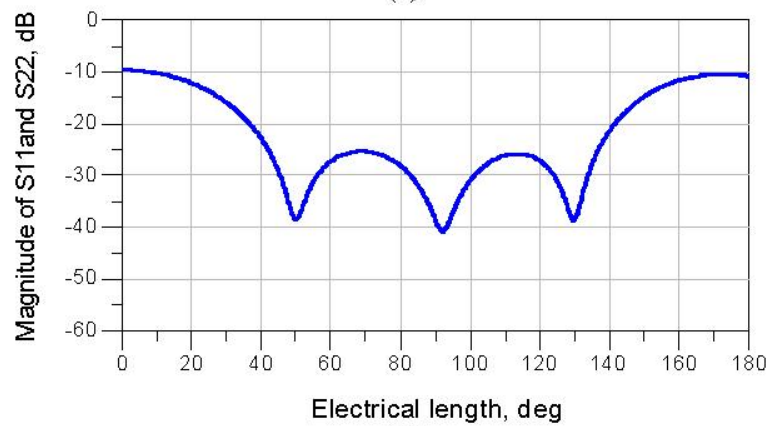

(b)

Fig. 8. Transformer based on two transmission lines (a) schematic, (b) calculated response.

Although the proposed structures are still quarterwavelength long, they provide almost three times wider operating frequency range in comparison to traditional quarter-wave transformer.

The considered examples demonstrate matching between resistive impedances. Complex impedance matching is possible by loading of the remaining terminals with complex loads (short/open stubs for example).

\section{ACKNOWLEDGEMENT}

The authors would like to acknowledge the partial financial support by the Danish Ministry of Research and Education.

\section{REFERENCES}

[1] Kian Sen Ang, Chee How Lee, and Yoke Choy Leong, "Analysis and design of coupled line impedance transformers," 2004 IEEE MTT-S Int. Microwave Symp. Dig., vol. 3, pp. 1951-1954, 2004.

[2] G. Jaworski, and V. Krozer, "Broadband matching of dual-linear polarization stacked probe-fed microstrip patch antenna," Electronics Letters, vol. 40, no. 4, pp. 221-222, 2004.

[3] S. P. Liu, "Planar transmission line transformer using coupled microstrip lines," IEEE MTT-S Int. Microwave Symp. Dig., vol. 2, pp. 789-792, 1998.

[4] R. Mongia, I. Bahl, P. Bhartia, RF and microwave coupled line circuits. Norwood: Artech House microwave library, 1999.

[5] V. K. Tripathi, "Asymmetric coupled transmission lines in an inhomogeneous medium," IEEE Trans. Microwave Theory \& Tech., vol. 23, no. 9, pp. 734-739, September 1975.

[6] D. Kajfez, S. Bokka, and C. E. Smith, "Asymmetric microstrip dc blocks with rippled response," 1987 IEEE MTT-S Int. Microwave Symp. Dig., pp. 301-303, 1981. 